\title{
Differential game theory with FPA optimization in multi-area power system
}

\author{
S. Khadarvali ${ }^{1}$, V. Madhusudhan ${ }^{2}$, R. Kiranmayi ${ }^{3}$ \\ 1,3 Department of Electrical and Electronics Engineering, Jawaharlal Nehru Technological University, India. \\ ${ }^{2}$ Department of Electrical and Electronics Engineering, VNR VJIET, India.
}

\begin{tabular}{l}
\hline Article Info \\
\hline Article history: \\
Received Aug 27, 2019 \\
Revised Nov 9, 2019 \\
Accepted Dec 2, 2019 \\
\hline
\end{tabular}

Keywords:

Flower pollination optimization Game theory

Load frequency control

Multi-area power system

Differential Game theory

\begin{abstract}
The distribution generation penetration increases due to the increased need of power. These penetration causes problem of frequency deviations. In this paper the in multi-area power system the combination of renewable energy resources is proposed. Here the area $1 \&$ area 2 are thermal power plant. Hydro power generation plant is taken in area-3 \& area-4. Renewable energy system is considered in area-5. Here the cyber security attack is taken as change in power in the entire area. This can make the power block out or wrong data entry. Here differential game theory-based problem formulation is done. The PI controller and differential game theory with flower pollination are compared for performance of fast response. The MATLAB $2017 \mathrm{~b}$ is used for building the areas and programming the algorithm.
\end{abstract}

This is an open access article under the CC BY-SA license.

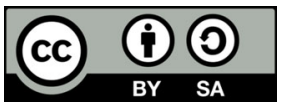

\section{Corresponding Author:}

S. Khadarvali,

Department of Electrical and Electronics Engineering,

Jawaharlal Nehru Technological University, Anantapuramu, India.

Email: khadar.v1@gmail.com

\section{INTRODUCTION}

The power system security is very important issue nowadays as the distribution generation penetration. Even small component failure brings more loss in power. This may affect the complete power system. The wide area control and monitoring produces the security in recent power systems. For that phasor measurement (PMU) units, circuit breakers and distributed generators uses the communication interfaces to make sure the system security. These security systems use cyber network as communication protocol there is a possibility of cyber-attack. Attackers inject false data injection as discussed in [1]. Eavesdropping estimate in [2] denies the service attack on the communication medium as in [3]. Analysis of risk is done in [4-6]. Recently the cyber-attack is done in the weaker system. The binary on-off pulses are created in [7] as cyberattack which can trip generators. False data injection makes the PMU to provide wrong data to power system control. This causes the central control unit to operate falsely. This causes blackouts and economical losses [8]. In [11] it is discussing that stealthy attack model which corrupts the smart circuit breakers.

The variation of renewable energy resources due to nature the power system must be capable of adjusting the output control of multi area power system. Generally, the power system consists of coal, hydro and gas. Many methods are used to control the power system in multi area [12,17-25]. In [13,14] keeping controls as same multi area with multiple sources are considered.

In this paper the flower pollination optimization algorithm is used for solving the game theory based objective functions. And here the distribution system is considered with multi area power system consist of hydro and steam unit. The changes in solar system creates the system to be weak. It makes the grid as prey 
for cyber-attacks. The new solution method gives the faster response in that condition compared to old methods. It reduces the vulnerability of attack.

This paper is organized like Controllert design in section 2, Flower pollination algorithm in section 3 , results and discussion in section 4 and last section with conclusion.

\section{CONTROLLER DESIGN}

According to the problem and situation many game theories are available. In this paper, "the nonzero-sum non-cooperative differential games" are used. "Nonzero sum" explains that it consists of more than two players, the sum of all the players' index of performance is zero or a constant. And collaboration between the players is difficult to enforce [15], henceforth the players are expected not to cooperate in order to minimize their individual performance index.

Take a system with $\mathrm{N}$ players defined by the linear differential equation as shown below

$$
\dot{x}(t)=A x(t)+\sum_{i=1}^{N} B_{i} u_{i}(t)
$$

where $x\left(t_{0}\right)=x_{0}$

where $x(t) \in R^{n \times t}$ is state varable vector

$u_{i}(t) \in R^{m_{i} \times 1}$ is a control strategy vector used by ith player

$A \in R^{n \times n}$

and

$B \in R^{n \times n}$ are constant real matrix

and each player desires to minimize his own quadratic simplicity.

Performance index, i.e., cost function. In this paper we adopt the following type cost function for

$J_{i}=\int_{t_{0}}^{\infty}\left\{x(t)^{T} Q_{i} x(t)+u_{i}(t)^{T} R_{i} u_{i}(t)\right\} d t$

where $Q_{i} \in R^{n \times n}$ is symmetric semi-positive definite

$R_{i} \in R^{m_{j} \times m_{j}}$ is symetric positive definite matrix

Assume current value of system state vector is available for all the players, then the constant linear feedback control strategy used by ith player can be expressed as

$$
\begin{aligned}
& u_{i}=F_{i} x \\
& F_{i} \in R^{m_{i} \times n} \\
& \text { where }\left(F_{1}, \ldots, F_{N}\right) \text { belongs to the set } F=\left\{F=\left(F_{1}, \ldots, F_{2}\right) \mid A+\sum_{i}^{N} B_{i} F_{i}\right\} \text { i s stable }
\end{aligned}
$$

\section{FLOWER POLLINATION ALGORITHM}

There are two important steps in this algorithm as it is given in [16], they are "global pollination and local pollination". The first step and flower constancy can be represented mathematically as

$$
x_{i}^{t+1}=x_{i}^{t}+L\left(x_{i}^{t}-g_{*}\right)
$$

where

$\mathrm{x}_{\mathrm{t}}^{i}-\quad$ is the pollen $\mathrm{i}$ or solution vector $\mathrm{x} \_\mathrm{i}$ at iteration $\mathrm{t}$,

$\mathrm{g}^{*}-$ is the current best solution found among all solutions at the current iteration,

$\mathrm{L}-$ is the strength of the pollination, which essentially is a step size.

Since insects may go a longer distance with various distances/steps, it uses a L'evy flight to mimic this characteristic professionally. So, draw $\mathrm{L}>0$ from a levy distribution as shown below,

$$
L \sim \frac{\lambda \Gamma(\lambda) \sin \left(\frac{\pi \lambda}{2}\right)\left(\frac{1}{s^{1+\lambda}}\right)}{\pi} \quad\left(s \gg s_{0} \gg 0\right)
$$


Pseudo code:

Objective $\min \mathrm{J}_{\mathrm{i}}(\mathrm{x}), \mathrm{x}=(\mathrm{x} 1, \mathrm{x} 2, \ldots, \mathrm{xd})$

Initializa a population of $n$ flowers/polln gametes with random solutions

Find the best solution $g_{-}$in the initial population

Define a switch probability $\mathrm{p} \in[0,1]$

while ( $\mathrm{t}<$ MaxGeneration)

for $\mathrm{i}=1: \mathrm{n}$ (all $\mathrm{n}$ flowers in the population)

if rand $<\mathrm{p}$,

Draw a (d-dimensional) step vector $\mathrm{L}$ which obeys a L'evy distribution

Global pollination via

$x_{i}^{t+1}=x_{i}^{t}+L\left(x_{i}^{t}-g_{*}\right)$

else

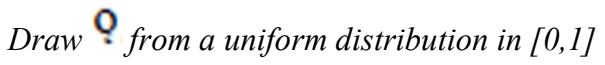

Randomly choos $\mathrm{j}$ and $\mathrm{k}$ among all solutions

Do local pollination via

$x_{i}^{t+1}=x_{i}^{t}+\varepsilon\left(x_{i}^{t}-x_{k}^{t}\right)$

End if

Evaluate new solutions

If new solutions are better, update them in the population

End for

Find the current best solution $g^{*}$

End while

\section{RESULTS AND DISCUSSION}

Scenario 1 is taken as renewable energy-four area system execution, scenario 2 is taken as four-area, two hydro \& two thermal system, scenario 3 is taken as five-area, where thermal, hydro and renewable energy are hybrid. The safety risk is formed at the renewable energy place due to the frequency change $\&$ power change are compared (which is otherwise known as weak grid condition). And it can be seen that differential game theory works stable even with cyber treat produced.

The different types of controllers are considered as different cases here. Case1 is works with PI controller, case 2 is works with Robust controller. Figure 1 shows the proposed test system with five-area including solar.

Figures $2,4,6,8,10 \& 12$ shows the tie line power of three separate scenarios and cases. Figures $3,5,7,9,11$ and 13 shows the frequency deviation of three separate scenarios and cases. Here it is shown that all the tie line power \& frequency are produced as zero which depicts the controllability. But the settling time is dissimilar for each scenarios and corresponding cases. The security threat is resolved and the system stability is achieved and it also shown in figures.

Int J Pow Elec \& Dri Syst Vol. 11, No. 1, Mar 2020 : $302-308$ 


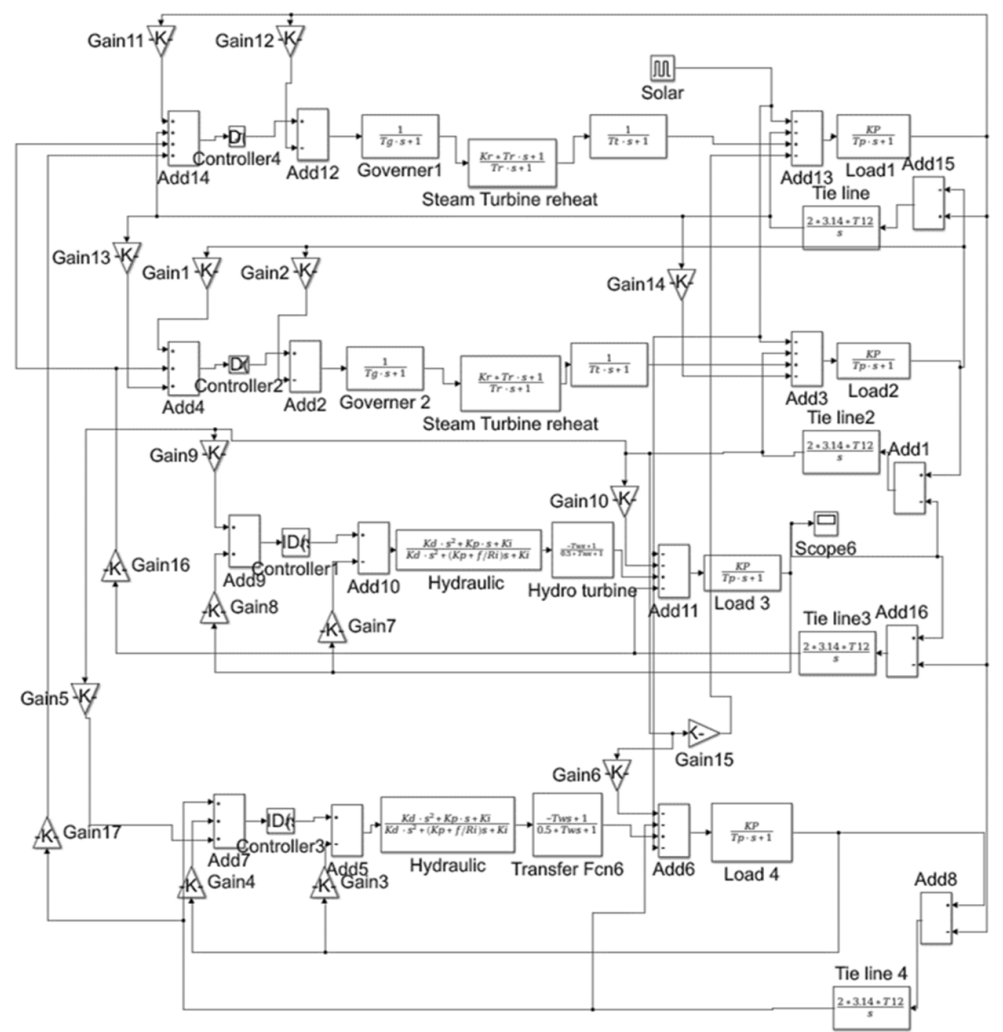

Figure 1. Proposed test system with 5 areas including solar

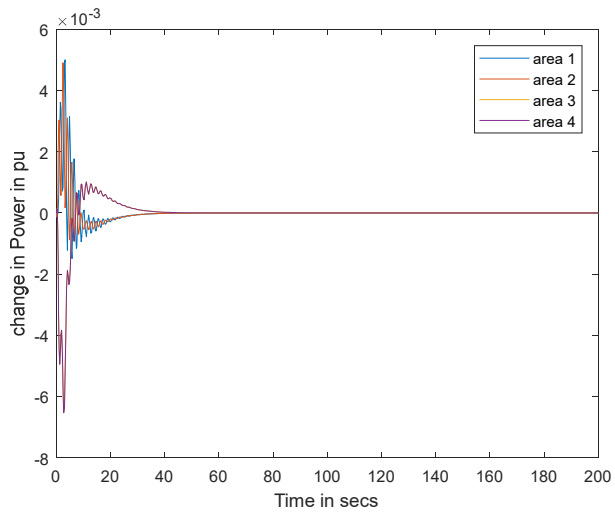

Figure 2. Tie line power in p.u with PI controller (scenario I-case I)

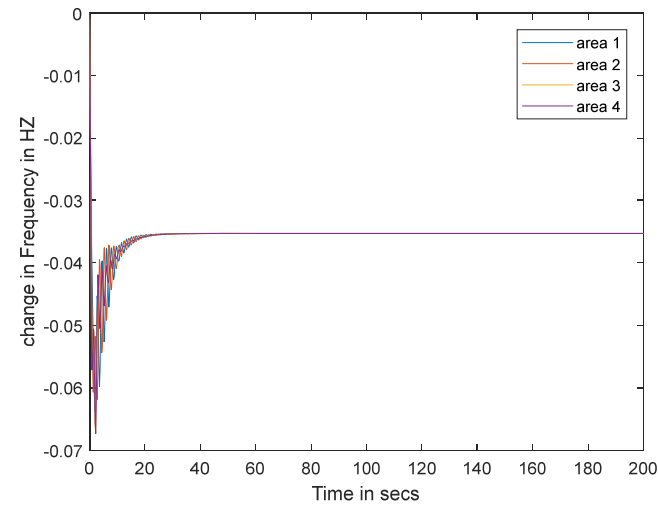

Figure 3. Frequency deviation in hz with PI controller (scenario 1-case I) 


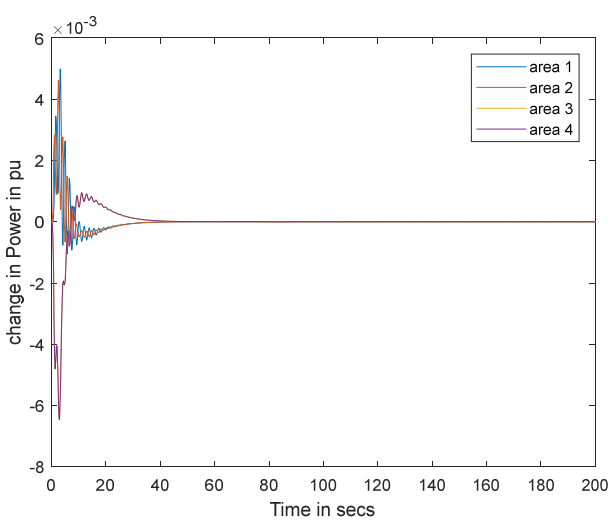

Figure 4. Tie line power in p.u with robust controller (scenario 1-case II)

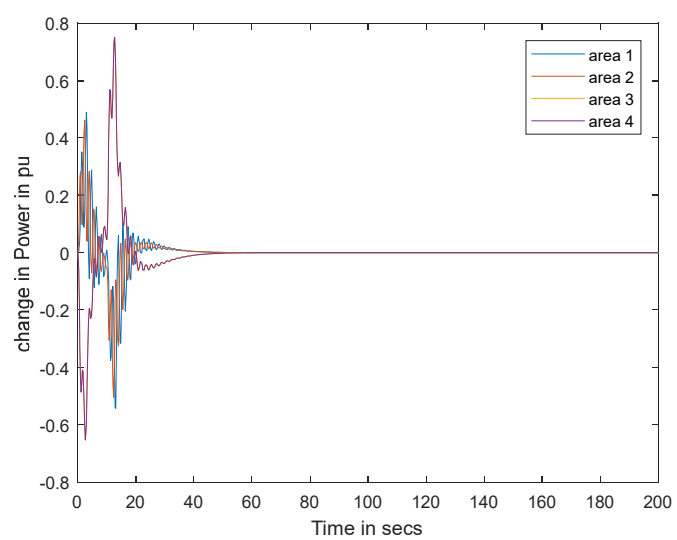

Figure 6. Tie line power in p.u with PI controller (scenario 2-case I)

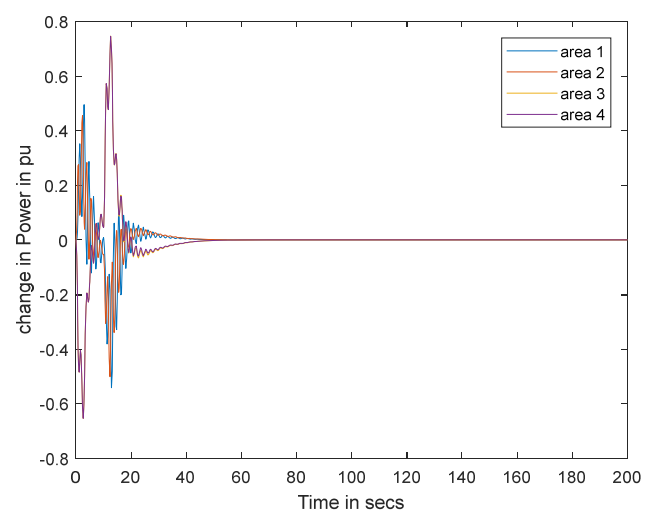

Figure 8 . Tie line power in p.u with robust controller (scenario 2- case II)

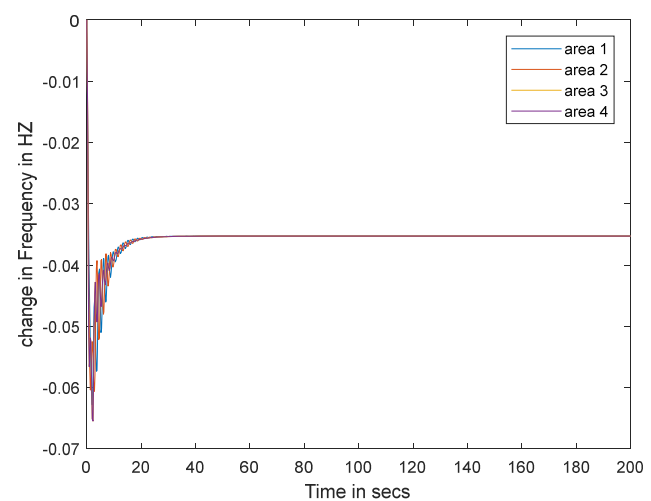

Figure 5. Frequency deviation in hz with robust controller (scenario 1-case II)

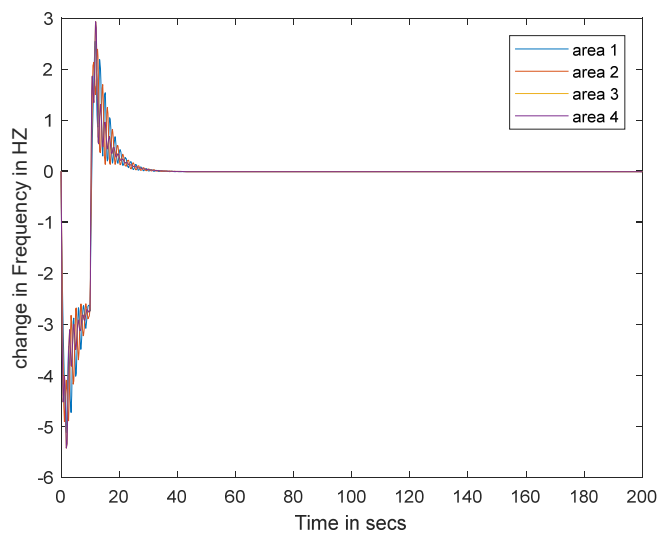

Figure 7. Frequency deviation in hz with PI controller (scenario 2-case I)

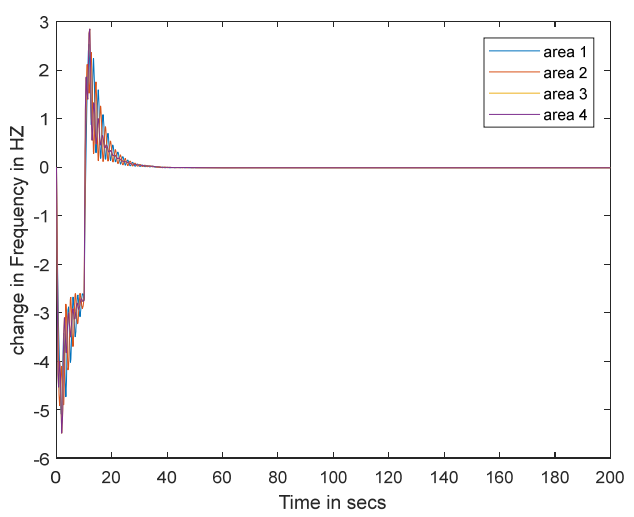

Figure 9. Frequency deviation in hz with robust controller (scenario 2- case II) 


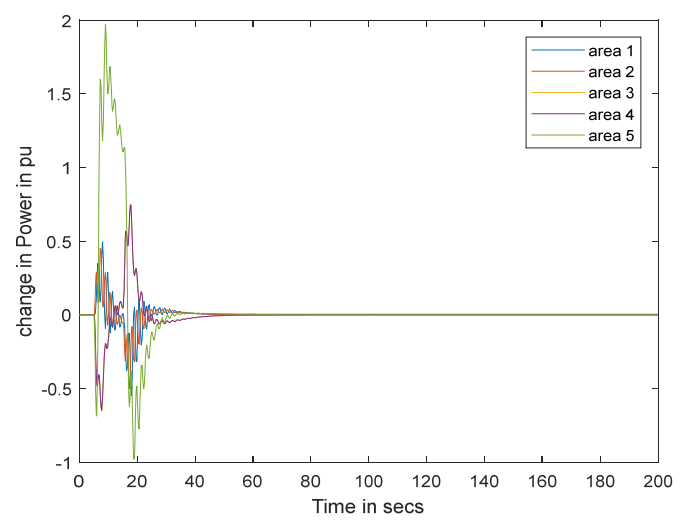

Figure 10. Tie line power in p.u with PI controller (scenario 3-case I)

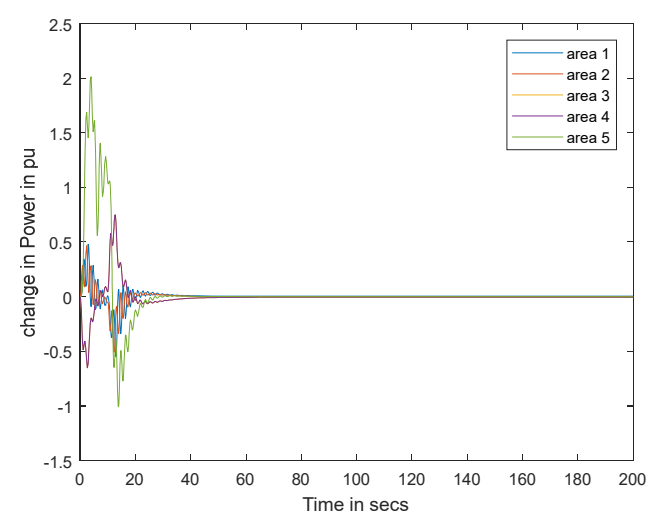

Figure 12. Tie line power in p.u with robust controller (scenario 3-case II)

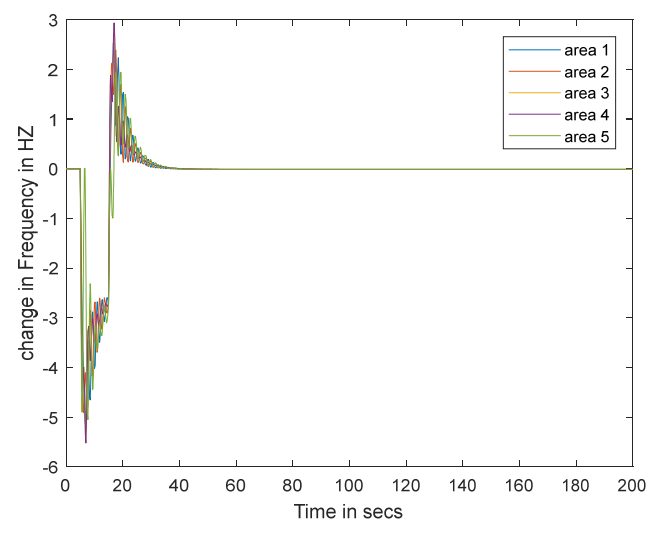

Figure 11. Frequency deviation in hz with PI controller (scenario 3-case I)

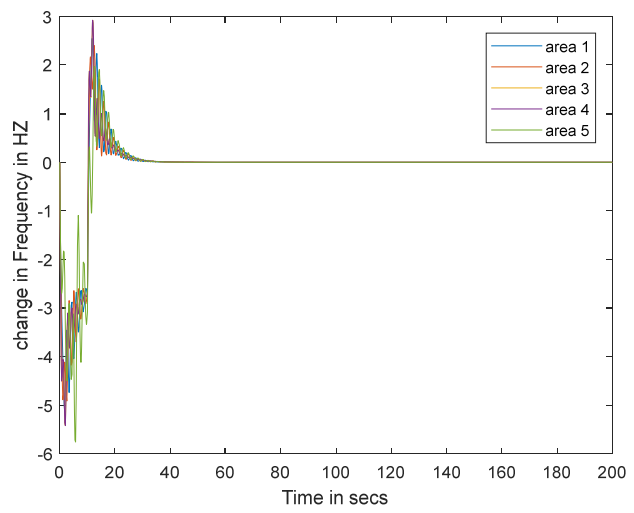

Figure 13. Frequency deviation in hz with robust controller (scenario 3-case II)

Table-1 shows the comparison of the results taken from all the three scenarios and the two cases studies. it shows that the case II of all the three scenarios performs better in rise time, settling time and peak time which are significant in the performance of the synamic operation of power system.

Table 1. Performance comparison table

\begin{tabular}{ccccccc}
\hline & \multicolumn{2}{c}{ Scenario 1 } & \multicolumn{2}{c}{ Scenario 2 } & \multicolumn{2}{c}{ Scenario 3 } \\
& PI & Robust & PI & Robust & PI & Robust \\
\hline RiseTime(s) & 4.1134 & 0.033927 & 0.4659 & 0.033927 & 2.0968 & 0.038871 \\
SettlingTime(s) & 21.502 & 10.611 & 26.421 & 8.1247 & 34.451 & 16.685 \\
SettlingMin & 0.14117 & 0.14117 & 0.046908 & 0.04677 & 0.063062 & 0.015 \\
SettlingMax & 0.26143 & 0.25803 & 21.327 & 21.415 & 25.779 & 26.341 \\
Overshoot & 85.183 & 82.769 & 45192 & 45407 & 40315 & $1.7524 \mathrm{e}+05$ \\
Undershoot & 0 & 0 & 0 & 0 & 0 & 0 \\
Peak & 0.26143 & 0.25803 & 21.327 & 21.415 & 25.779 & 26.341 \\
PeakTime (s) & 5.6118 & 2.3388 & 3.3785 & 1.3073 & 9.5024 & 3.1117 \\
\hline
\end{tabular}

\section{CONCLUSION}

The PI controller and robust control using differential game theory with Flower pollination algorithm is used to solve the problem of security attack created at the multi-area power system. The performance like settling time rise time and peak time shows the robustness of the new control system and it is depicted that in all the scenarios case II, the stability is attained in smaller time compared to case I. So, the controller proves that the multi-area with renewable resources stable even in cyber-attack. 


\section{REFERENCES}

[1] Y. Liu, P. Ning, and M. K. Reiter, "False data injection attacks against state estimation in electric power grids," in Proc. 16th ACM Conf. Comput. Commun. Security, Chicago, IL, USA, pp. 21-32, 2009.

[2] M. El-Halabi, A. Farraj, H. Ly, and D. Kundur, "A distortion-theoretic perspective for redundant metering security in smart grid," in Proc. IEEE Can. Conf. Elect. Comput. Eng. (CCECE), Montreal, Canada, Apr./May, pp. 1-5, 2012.

[3] D. Kundur, "Power system reliability, security and stability, class notes for ECE1518: Seminar in identity, privacy and security," Dept. Elect. Comput. Eng., Univ. Toronto, Toronto, ON, Canada, 2014.

[4] S. Sridhar, A. Hahn, and G. Manimaran, "Cyber-physical security for electric power grid," Proc. IEEE, vol. 100, no. 1, pp. 210-224, Jan. 2012

[5] A. Hahn and G. Manimaran, "Cyber attack exposure evaluation framework for the smart grid," IEEE Trans. Smart Grid, Vol. 2, No. 4, pp. 835-843, Dec. 2011

[6] R. P. Rezaei, P. Hines, and M. Eppstein, "Estimating cascading failure risk with random chemistry," IEEE Trans. Power Syst., Vol. 30, No. 5, pp. 2726-2735, Sep. 2015

[7] S. Liu, B. Chen, T. Zourntos, D. Kundur, and K. Butler-Purry, "A coordinated multi-switch attack for cascading failures in smart grid," IEEE Trans. Smart Grid, vol. 5, no. 3, pp. 1183-1195, May 2014.

[8] T. Liu et al., "Abnormal traffic-indexed state estimation: A cyber- physical fusion approach for smart grid attack detection," Future Gener.Comput. Syst., vol. 49, pp. 94-103, Aug. 2015.

[9] M. Esmalifalak, L. Liu, N. Nguyen, R. Zheng, and Z. Han, "Detecting stealthy false data injection using machine learning in smart grid," IEEE Syst. J., DOI: 10.1109/JSYST.2014.2341597.

[10] M. Esmalifalak, Z. Han, and L. Song, "Effect of stealthy bad data injection on network congestion in market-based power system," in Proc. IEEE Wireless Commun. Netw. Conf., Shanghai, China, 2012, pp. 2468-2472.

[11] S. Liu, B. Chen, D. Kundur, T. Zourntos, and K. Butler-Purry, "Progressive switching attacks for instigating cascading failures in smart grid," in Proc. IEEE Power Energy Soc. Gen. Meeting, Vancouver, BC, Canada, 2013, pp. $1-5$.

[12] H. Shayeghi, H. A. Shayanfar and A. Jalili, "Load frequency control strategies: a state-of-the-art survey for the researcher," Energy Conversion and Management, vol. 50, no. 2, pp. 344-353, Feb. 2011.

[13] K. S. S. Ramakrishna1, P. Sharma and T. S. Bhatti, "Automatic generation control of interconnected power system with diverse sources of power generation," International Journal of Engineering Science and Technology, vol. 2, no. 5, pp. 51-65, 2010.

[14] K. P. S. Parmara, S. Majhi and D. P. Kothari, "Load frequency control of a realistic power system with multisource power generation," Electrical Power and Energy Systems, vol. 42, no. 1, pp. 426-433, Nov. 2012.

[15] J. C. Engwerda, LQ dynamic optimization and differential games. New York: Wiley, 2005

[16] Xin-she Yang, "Flower Pollination algorithm for Global Optimization", Unconventional Computation and Natural Computation 2012, Lecture Notes in Computer Science, Vol. 7445, pp. 240-249, 2012.

[17] N. E. Y. Kouba, M. Menaa, M. Hasni and M. Boudour, "Load Frequency Control in multi-area power system based on Fuzzy Logic-PID Controller," 2015 IEEE International Conference on Smart Energy Grid Engineering (SEGE), Oshawa, ON, 2015, pp. 1-6.

[18] BAO, YQ., LI, Y., WANG, B. et al. "Demand response for frequency control of multi-area power system" J. Mod. Power Syst. Clean Energy (2017) 5: 20.

[19] Rabindra Kumar Sahu, Tulasichandra Sekhar Gorripotu, Sidhartha Panda, "Automatic generation control of multiarea power systems with diverse energy sources using Teaching Learning Based Optimization algorithm", Engineering Science and Technology, an International Journal, Vol. 19, No. 1, pp. 113-134, 2016.

[20] Zeng, Guo-Qiang \& Xie, Xiao-Qing \& Chen, Min-Rong. "An Adaptive Model Predictive Load Frequency Control Method for Multi-Area Interconnected Power Systems with Photovoltaic Generations" Energies. Vol. 10, 2017. 10.3390/en10111840.

[21] J. Devendra Kumar, Mr.M. Santosh Kiran, "Multi Area Load Frequency Control in Power Systems via Internal Model Control Scheme using Model-Order Reduction", International Journal of Advanced Research in Electrical, Electronics and Instrumentation Engineering, Vol. 2, Issue 12, December 2013, pages 6443-6454.

[22] Isha Garg, "Multi-Area Load Frequency Control Implementation in Deregulated Power System", International Journal of Soft Computing and Engineering (IJSCE), vol. 2, no. 2, 2012.

[23] Gomaa Haroun AH, Li YY, "A novel optimized hybrid fuzzy logic intelligent PID controller for an interconnected multi-area power system with physical constraints and boiler dynamics", ISA Trans. Vol 71 (Pt 2) pp. 364-379, 2017.

[24] Topno, Pretty \& Chanana, Saurabh, "Load frequency control of a two-area multi-source power system using a tilt integral derivative controller" Journal of Vibration and Control. Vol 24, 2016.

[25] H.D. Mathur and H.V. Manjunath, "Frequency stabilization using fuzzy logic-based controller for multi-area power system", The South Pacific Journal of Natural Science, vol. 4, pp. 22-30, 2007. 